\title{
Cancer stem cells, tumor dormancy, and metastasis
}

\section{Purvi Patel and Emily I. Chen*}

Department of Pharmacological Sciences, Stony Brook University, Stony Brook, NY, USA

\section{Edited by: \\ Annette Meeson, Newcastle \\ University, UK}

\section{Reviewed by:}

Anthony Rhodes, University of the

West of England, UK

Federica Barbieri, University of

Genoa, Italy

\section{${ }^{*}$ Correspondence:}

Emily I. Chen, Department of Pharmacological Sciences, Stony Brook University, BST-125, Stony Brook, NY 11794-8651, USA

e-mail:emily@pharm.stonybrook.edu

\begin{abstract}
Tumor cells can persist undetectably for an extended period of time in primary tumors and in disseminated cancer cells. Very little is known about why and how these tumors persist for extended periods of time and then evolve to malignancy. The discovery of cancer stem cells (CSCs) in human tumors challenges our current understanding of tumor recurrence, drug resistance, and metastasis, and opens up new research directions on how cancer cells are capable of switching from dormancy to malignancy. Although overlapping molecules and pathways have been reported to regulate the stem-like phenotype of CSCs and metastasis, accumulated evidence has suggested additional clonal diversity within the stem-like cancer cell subpopulation. This review will describe the current hypothesis linking CSCs and metastasis and summarize mechanisms important for metastatic CSCs to re-initiate tumors in the secondary sites. A better understanding of CSCs' contribution to clinical tumor dormancy and metastasis will provide new therapeutic revenues to eradicate metastatic tumors and significantly reduce the mortality of cancer patients.
\end{abstract}

\footnotetext{
Keywords: cancer stem cells, metastasis, tumor dormancy, stem-like subpopulations, disseminated CSCs, EMTMET cooperativity
}

\section{INTRODUCTION}

Solid tumors account for the major cancer burden, and epithelial cancers arising in breast, lung, colon, prostate, and ovary comprise approximately $80 \%$ of all cancers (Visvader and Lindeman, 2008). However, over $90 \%$ of mortality in cancer patients is attributed to the subsequent spread of cancer cells to distant tissues (Weigelt et al., 2005; Jemal et al., 2006; Steeg, 2006). In patients, the threat of tumor can return after chemotherapy and radiation remains terrifying and painfully real. In breast cancer, for example, metastasis can occur after decades of apparent disease-free period prior to the development of distant metastasis (Meltzer, 1990; Uhr et al., 1997). This phenomenon is referred to as clinical tumor dormancy and is frequently observed in cancer patients. Both clinical observations and experimental models have revealed that cancer patients may have hundreds to thousands of disseminated cancer cells detectable in circulation but only a small portion of the disseminated cancer cells progress to form clinically overt metastases (Tarin et al., 1984; Weiss, 1990, 1992).

Metastasis is a multi-step process. It is well recognized that cancer cells capable of metastasizing acquire epithelial-mesenchymal transition (EMT)-like phenotype allowing them to disseminate from the primary tumor and intravasate into the circulation. In circulation, these disseminated cancer cells have to survive and then eventually extravasate into foreign tissues. Finally, few of these cells will adapt in the microenvironment and form macrometastases. Experimental evidence has demonstrated that early step of metastasis (intravasate, survival, arrest, and extravasation) can be highly efficient (Cameron et al., 2000; Suzuki et al., 2006). However, only a small subset of these cells $(\sim 2 \%)$ can initiate growth as micrometastases, and an even smaller fraction of these cells $(\sim 0.02 \%)$ are able to persist and form macrometastases (Cameron et al., 2000; Chambers et al., 2000, 2001; Suzuki et al., 2006). Therefore, the later steps of metastasis appear to be the most critical steps for metastatic dormancy. In spite of the clinical importance of metastasis, the mechanisms underlying the process of dormancy and outgrowth of macrometastases remain poorly understood.

Accumulating evidence suggests that a subpopulation of cancer cells exhibit stem-like properties and is capable of tumor initiation, invasive growth, and disseminating to distant organs (Reya et al., 2001; Li et al., 2007; Liu et al., 2010; Marotta and Polyak, 2009; O'Brien et al., 2009). These cancer stem cells (CSCs) have the ability to self-renew to give rise to other tumorigenic cells, as well as undergo differentiation to give rise to the phenotypically diverse non-tumorigenic cancer cells. Several characteristics of CSCs, including the phenotypic plasticity, make them more likely to succeed in the later steps of metastasis. This review will focus on specific stem cell features of CSCs relating to cancer metastasis and implications of CSC theory on treatment strategies against metastasis.

\section{STEM CELL CHARACTERISTICS RELATING TO TUMOR DISSEMINATION}

It is well recognized that some cancer cells are capable of undergoing an EMT-like transformation and develop a migratory and invasive phenotype to detach from the primary tumor (Kang and Massague, 2004; Micalizzi et al., 2010; Gomes et al., 2011; Nauseef and Henry, 2011; Said and Williams, 2011; Yao et al., 2011). EMT is a biologic process that allows epithelial cells to undergo multiple biochemical changes that acquire a mesenchymal cell phenotype with an enhanced migratory capacity, invasiveness, elevated resistance to apoptosis, and increased production of ECM components (Kalluri, 2009; Yilmaz and Christofori, 2009). CSCs have been hypothesized to be the disseminating subpopulation and supported by accumulating evidence that CSCs also express EMT markers, and more importantly, induction of EMT in transformed epithelial cells promotes the generation of CSCs 
(Yang etal., 2004; Mani etal., 2008; Floor etal., 2011; Jordan etal., 2011; Wu, 2011; Wu and Yang, 2011; Krantz etal., 2012). For example, in colon cancer, nuclear accumulation of $\beta$-catenin, the feature of Wnt signaling activation and stem cell signaling, is found at the invasive front of the primary tumor (Fodde and Brabletz, 2007). Stem-like cells isolated from normal mammary glands and breast tumors also express EMT markers (Damonte etal., 2007; Mani etal., 2008). Stem-like breast cancer cells isolated from exposing breast cancer cells to cycles of hypoxia and reoxygenation also show up-regulation of several EMT-inducing factors (Louie et al., 2010). Overexpression of EMT-inducing transcription factors such as Snail or Twist, in transformed mammary epithelial cells (HMLEM) increased tumor-initiating frequency in immune-deficient mice (Mani et al., 2008). Similar to the embryonic programming of EMT, activation of EMT signaling in CSCs is also regulated by a reciprocal feedback loop between the ZEB family of EMT inducers and miR200 family of EMT suppressors (Hurteau et al., 2007; Burk et al., 2008; Gregory et al., 2008; Korpal et al., 2008; Park et al., 2008) as well as a second reciprocal feedback loop with EMT suppressor, miR-34, and EMT inducer, Snail (Kim et al., 2011; Siemens et al., 2011). Together, these observations lend support to the model of migrating CSCs that confer the plasticity of switching between cellular states and detaching efficiently from the primary tumor.

\section{STEM CELL CHARACTERISTICS RELATING TO DORMANCY AND METASTASIS}

In addition to the self-renewing and EMT phenotypes, CSCs also exhibit other stem cell properties that are beneficial for them to adapt in the foreign microenvironment and eventually form clinical overt metastasis. It is apparent that several unique properties necessary for ensuring long life span of normal stem cells may contribute to protection of CSCs in the adverse microenvironment. For example, normal stem cells have increased capacity for DNA repair and express higher levels of anti-apoptotic proteins than differentiated cells (Cairns, 2002; Potten et al., 2002; Wang et al., 2003; Park and Gerson, 2005; Shinin et al., 2006). After disseminated cancer cells arrive at the foreign microenvironment, the suppressive target organ can form a barrier to halt the progression of metastasis and induce dormancy. The enhanced anti-apoptotic and DNA repair capability of CSCs could increase the survival of CSCs for a long period of time under metabolic and/or other environmental stress (e.g., hypoxia) in the target organ and allow them to find adaptive solutions.

Evidence supporting this model comes from studies showing CSCs have increased drug resistance capacity. For example, it has been shown that stem-like subpopulation of cancer cells express high levels of ATP-binding cassette (ABC) transporters that can actively efflux drugs and shield them from the adverse effects of chemotherapeutic insult (Pardal et al., 2003; Lou and Dean, 2007; Dean, 2009; Donnenberg et al., 2009; Ding et al., 2010; Moitra et al., 2011). In addition to an increased drug efflux capacity, CSCs also exhibit intrinsic resistance to apoptosis. For instance, autocrine production of cytokines such as IL- 4 has been shown to increased anti-apoptotic proteins and induces resistance to therapy-induced cytotoxicity in different cancer types
(Conticello et al., 2004). For patients with colon cancer, IL-4 antagonist was shown to strongly enhance the anti-tumor efficacy of conventional chemotherapeutic drugs through selective sensitization of colon CSCs that are CD133+ (Todaro et al., 2007). There is also growing evidence that CSCs are inherently resistant to radiation (Koepke, 2006; Rich, 2007; Debeb et al., 2009; Pajonk et al., 2010; Croker and Allan, 2012; D'Andrea, 2012). For example, the effectiveness of radiotherapy is mediated by the induction of reactive oxygen species (ROS) in cancer cells. However, it has been found that both human and mouse mammary CSCs contain lower ROS levels than more differentiated tumor cells and accumulate less DNA damage upon radiation (Diehn et al., 2009). Lower ROS levels in CSCs appear to result from increased expression of free radical scavenging systems (Diehn et al., 2009; Wang et al., 2010; Kobayashi and Suda, 2012; Shi et al., 2012). Together, the experimental evidence is consistent with clinical observations that drug resistance is seemingly higher in metastatic disease. More importantly, the inherent feature of drug resistance in CSCs could activate stress responses to protect them from growth-suppressing conditions in the target organ microenvironment and allow them to persist in foreign tissues for a long period of time.

\section{STEM CELL CHARACTERISTICS RELATING TO THE FORMATION OF CLINICALLY OVERT METASTASIS}

It has been observed that a high proportion of distant metastases are differentiated and in some cases metastases can show a greater degree of cellular differentiation than the primary tumors. For example, increased E-cadherin expression in metastases compared to the primary tumors has been reported in human patient specimens (Oka etal., 1993; Kowalski et al., 2003; Chao et al., 2010). Furthermore, the importance of epithelial phenotype in the formation of secondary tumors has been demonstrated in different metastasis models, including bladder cancer (Chaffer et al., 2005, 2006, 2007), prostate cancer (Oltean et al., 2006; Yates et al., 2007), colorectal cancer (Vincan et al., 2007a,b), and breast cancer (Tsuji et al., 2008, 2009; Chao et al., 2010). Hence, both clinical and experiment evidence points to the necessity of disseminated cancer cells undergoing a mesenchymal to epithelial reverting transition (MET) in the secondary microenvironment to form macrometastases. Consequently, it has been proposed that metastatic cancer cells possess the phenotypic plasticity and acquired EMT-like phenotype for disseminating from the primary tumor and subsequently, a second transition from the EMT-like to MET-like state occurs to facilitate the formation of metastatic tumors at target organs (Brabletz, 2012). However, it is unclear whether EMT/stem-like cancer cells (CSCs) cooperate with nonEMT cancer cells for successful colonization at target organs or CSCs maintain a fused cell phenotype with incomplete or mixed epithelial characteristics that can be used to initiate the subsequent adaptation in the foreign microenvironment. For example, Tsuji et al. (2008) showed that cancer cells with an induced EMT state increase invasiveness locally in the primary tumor but fail to promote distant colonization when introduced in circulation. Also, co-culture of hepatocytes and EMT-like prostate cancer cells resulted in up-regulation of E-cadherin in these cells (Yates et al., 2007). We also found that brain metastatic breast cancer cells 
utilize oxidative metabolism that is similar to the normal epithelial cells instead of the glycolytic metabolism utilized by rapidly proliferating EMT-like breast cancer cells (Chen et al., 2007).

To complicate matters further, the metastatic niche can also supplies extrinsic factors that can further influence the proliferation and cellular states of metastatic cancer cells. Proliferation of disseminated CSCs in the targeted organs can be regulated by growth-suppressing and growth-stimulating factors secreted by or into the microenvironment. For example, a single dormant cancer cell or a dormant micrometastasis can turn into clinically detectable metastasis through an increased secretion of angiogenic factors in the metastatic niche to promote the recruitment and formation of new blood vessels (angiogenesis; Takahashi and Mai, 2005; Gao etal., 2008; Raza et al., 2010; Saharinen et al., 2011; Garcia and Kandel, 2012). It has been reported that CSCs promote tumor angiogenesis by actively secreting angiogenic factors such as vascular endothelial growth factor (VEGF; Bao et al., 2006; Seton-Rogers, 2011). In addition to secreted factors, cell-cell interactions in the microenvironment can also mediate protection of disseminated CSCs in the metastatic niche. For instance, it has been shown that mesenchymal stem cells (MSCs) induce the production of regulatory $\mathrm{T}$ cells $\left(\mathrm{T}_{\text {regs }}\right)$ when co-cultured with breast cancer cells allowing for immune subversion of breast cancer cells in the bone marrow (Patel et al., 2010). Also, it has been shown that bone marrow-derived MSCs could promote the expansion of CSC population in the co-culture system and accelerate tumor growth in the xenograft mouse model (Liu etal., 2011). Therefore, interactions between disseminated CSCs and tumor-associated MSCs may play a role in preventing the elimination of cancer cells by normal anti-tumor immune responses in the metastatic microenvironment.

\section{THERAPEUTIC CONSEOUENCES AND PERSPECTIVES OF MIGRATING CANCER STEM CELLS}

The presence of a distinct population with stem cell characteristics among disseminated and circulating cancer cells has an important clinical relevance in metastasis formation and recurrence, but more importantly for their role in resistance to conventional

\section{REFERENCES}

Bao, S., Wu, Q., Sathornsumetee, S., Hao, Y., Li, Z., Hjelmeland, A. B., et al. (2006). Stem cell-like glioma cells promote tumor angiogenesis through vascular endothelial growth factor. Cancer Res. 66, 7843-7848.

Brabletz, T. (2012). To differentiate or not - routes towards metastasis. Nat. Rev. Cancer 12, 425-436.

Burk, U., Schubert, J., Wellner, U., Schmalhofer, O., Vincan, E., Spaderna, S., etal. (2008). A reciprocal repression between $\mathrm{ZEB} 1$ and members of the miR-200 family promotes EMT and invasion in cancer cells. EMBO Rep. 9, 582-589.

Cairns, J. (2002). Somatic stem cells and the kinetics of mutagenesis and

anti-cancer therapy. The stem-like properties of CSCs have been shown to be advantageous in protecting them from environmental and genotoxic stresses. The observed phenotypic plasticity of CSCs and their inherent anti-apoptotic ability can potentially render them capable of navigating through the entire metastatic cascade. It is apparent that current therapeutic strategies fail to account for potential molecular and proliferative differences between different subpopulations of tumor cells. This may explain why the majority of therapies fail in treating patients with metastatic diseases. CSC model also has significant implications for cancer progression. It has been observed in clinics that remission generally becomes more difficult to achieve with each relapse, and the diagnosis of metastatic disease significantly reduces patient survival. This suggests that the cancer cell population becomes increasingly aggressive over time, perhaps reflecting the selectively killing of the non-tumorigenic cells and enriching for the inherently therapeutic resistant CSCs in the metastatic tumor. Indeed, accumulating experimental evidence has confirmed this suspicion and demonstrated the potential benefit of selectively targeting the stem-like subpopulation in preventing cancer progression (Gupta et al., 2009; Newman et al., 2012). However, alternative evidence opposing CSC model also exists suggesting that CSCs may not be the only cell type to sustain tumor growth and generate metastasis (Kelly et al., 2007; Yoo and Hatfield, 2008). Despite of the controversial origin or source of stem-like subpopulations, it is clear that a better definition and understanding of CSCs in clinical tumor dormancy as well as cancer relapse will have a significant impact on the mortality of patients with advanced stage of cancer.

\section{ACKNOWLEDGMENTS}

We thank members of our laboratory and our collaborators for their research work and helpful discussion. Wherever possible we refer the reader to the citations of primary papers. We apologize to the authors whose work we could not cite directly because of space restrictions. Our studies mentioned in this review were supported by the Susan Komen Breast Cancer Foundation and the Mary Anita Conroy Memorial Breast Cancer Fund from Manhasset Women's Coalition Against Breast Cancer.

M., Thompson, E. W., et al. (2005). Upregulated MT1-MMP/TIMP-2 axis in the TSU-Pr1-B1/B2 model of metastatic progression in transitional cell carcinoma of the bladder. Clin. Exp. Metastasis 22, 115-125.

Chaffer, C. L., Thompson, E. W., and Williams, E. D. (2007). Mesenchymal to epithelial transition in development and disease. Cells Tissues Organs 185, 7-19.

Chambers, A. F., MacDonald, I. C. Schmidt, E. E., Morris, V. L., and Groom, A. C. (2000). Clinical targets for anti-metastasis therapy. Adv. Cancer Res. 79, 91-121.

Chambers, A. F., Naumov, G. N., Varghese, H. J., Nadkarni, K. V., MacDonald, I. C., and Groom, A. C.
(2001). Critical steps in hematogenous metastasis: an overview. Surg. Oncol. Clin. N. Am. 10, 243-255, vii.

Chao, Y. L., Shepard, C. R., and Wells, A. (2010). Breast carcinoma cells re-express E-cadherin during mesenchymal to epithelial reverting transition. Mol. Cancer 9, 179.

Chen, E. I., Hewel, J., Krueger, J. S., Tiraby, C., Weber, M. R., Kralli, A., et al. (2007). Adaptation of energy metabolism in breast cancer brain metastases. Cancer Res. 67, 14721486.

Conticello, C., Pedini, F., Zeuner, A., Patti, M., Zerilli, M., Stassi, G., et al. (2004). IL-4 protects tumor cells from anti-CD95 and chemotherapeutic agents via up-regulation of 
antiapoptotic proteins. J. Immunol. 172, 5467-5477.

Croker, A. K., and Allan, A. L. (2012). Inhibition of aldehyde dehydrogenase (ALDH) activity reduces chemotherapy and radiation resistance of stem-like ALDHhiCD44+ human breast cancer cells. Breast Cancer Res. Treat. 133, 75-87.

Damonte, P., Gregg, J. P., Borowsky, A. D., Keister, B. A., and Cardiff, R. D. (2007). EMT tumorigenesis in the mouse mammary gland. Lab. Invest. 87, 1218-1226.

D’Andrea, F. P. (2012). Intrinsic radiation resistance of mesenchymal cancer stem cells and implications for treatment response in a murine sarcoma model. Dan. Med. J. 59, B4388.

Dean, M. (2009). ABC transporters, drug resistance, and cancer stem cells. J. Mammary Gland Biol. Neoplasia 14, 3-9.

Debeb, B. G., Xu, W., and Woodward, W. A. (2009). Radiation resistance of breast cancer stem cells: understanding the clinical framework. J. Mammary Gland Biol. Neoplasia 14, 11-17.

Diehn, M., Cho, R. W., Lobo, N. A., Kalisky, T., Dorie, M. J., Kulp, A. N., et al. (2009). Association of reactive oxygen species levels and radioresistance in cancer stem cells. Nature 458 , 780-783.

Ding, X. W., Wu, J. H., and Jiang, C. P. (2010). ABCG2: a potential marker of stem cells and novel target in stem cell and cancer therapy. Life Sci. 86, 631-637.

Donnenberg, V. S., Meyer, E. M., and Donnenberg, A. D. (2009). Measurement of multiple drug resistance transporter activity in putative cancer stem/progenitor cells. Methods Mol. Biol. 568, 261-279.

Floor, S., van Staveren, W. C., Larsimont, D., Dumont, J. E., and Maenhaut, C. (2011). Cancer cells in epithelial-to-mesenchymal transition and tumor-propagating-cancer stem cells: distinct, overlapping or same populations. Oncogene 30 , 4609-4621.

Fodde, R., and Brabletz, T. (2007). Wnt/beta-catenin signaling in cancer stemness and malignant behavior. Curr. Opin. Cell Biol. 19, 150-158.

Gao, D., Nolan, D. J., Mellick, A. S., Bambino, K., McDonnell, K., and Mittal, V. (2008). Endothelial progenitor cells control the angiogenic switch in mouse lung metastasis. Science 319, 195-198.

Garcia, A., and Kandel, J. J. (2012). Notch: a key regulator of tumor angiogenesis and metastasis. Histol. Histopathol. 27, 151-156.
Gomes, L. R., Terra, L. F., Sogayar, M. C., and Labriola, L. (2011). Epithelial-mesenchymal transition: implications in cancer progression and metastasis. Curr. Pharm. Biotechnol. 12, 1881-1890.

Gregory, P. A., Bert, A. G., Paterson, E. L., Barry, S. C., Tsykin, A., Farshid, G., et al. (2008). The miR-200 family and miR-205 regulate epithelial to mesenchymal transition by targeting ZEB1 and SIP1. Nat. Cell Biol. 10 593-601.

Gupta, P. B., Onder, T. T., Jiang, G., Tao, K., Kuperwasser, C., Weinberg, R. A., et al. (2009). Identification of selective inhibitors of cancer stem cells by high-throughput screening. Cell 138, 645-659.

Hurteau, G. J., Carlson, J. A., Spivack, S. D., and Brock, G. J. (2007). Overexpression of the microRNA hsa-miR$200 \mathrm{c}$ leads to reduced expression of transcription factor 8 and increased expression of E-cadherin. Cancer Res. 67, 7972-7976.

Jemal, A., Siegel, R., Ward, E., Murray, T., Xu, J., Smigal, C., et al. (2006). Cancer statistics, 2006. CA Cancer J. Clin. 56, 106-130.

Jordan, N. V., Johnson, G. L., and Abell, A. N. (2011). Tracking the intermediate stages of epithelialmesenchymal transition in epithelial stem cells and cancer. Cell Cycle 10, 2865-2873.

Kalluri, R. (2009). EMT: when epithelial cells decide to become mesenchymallike cells. J. Clin. Invest. 119, 14171419.

Kang, Y., and Massague, J. (2004) Epithelial-mesenchymal transitions: twist in development and metastasis. Cell 118, 277-279.

Kelly, P. N., Dakic, A., Adams, J. M., Nutt, S. L., and Strasser, A. (2007). Tumor growth need not be driven by rare cancer stem cells. Science 317, 337

Kim, N. H., Kim, H. S., Li, X. Y., Lee, I., Choi, H. S., Kang, S. E., et al. (2011). A p53/miRNA-34 axis regulates Snail1-dependent cancer cell epithelial-mesenchymal transition. J. Cell Biol. 195, 417-433.

Kobayashi, C. I., and Suda, T. (2012). Regulation of reactive oxygen species in stem cells and cancer stem cells. J. Cell. Physiol. 227, 421-430.

Koepke, T. (2006). Cancer stem cells linked to radiation resistance. Cancer Biol. Ther. 5, 1428.

Korpal, M., Lee, E. S., Hu, G., and Kang, Y. (2008). The miR-200 family inhibits epithelial-mesenchymal transition and cancer cell migration by direct targeting of E-cadherin transcriptional repressors ZEB1 and
ZEB2. J. Biol. Chem. 283, 14910 14914.

Kowalski, P. J., Rubin, M. A., and Kleer, C. G. (2003). E-cadherin expression in primary carcinomas of the breast and its distant metastases. Breas Cancer Res. 5, R217-R222.

Krantz, S. B., Shields, M. A., DangiGarimella, S., Munshi, H. G., and Bentrem, D. J. (2012). Contribution of epithelial-to-mesenchymal transition and cancer stem cells to pancreatic cancer progression. J. Surg. Res. 173, 105-112.

Li, F., Tiede, B., Massague, J., and Kang, Y. (2007). Beyond tumorigenesis: cancer stem cells in metastasis. Cell Res. 17, 3-14.

Liu, H., Patel, M. R., Prescher, J. A., Patsialou, A., Qian, D., Lin, J., et al. (2010). Cancer stem cells from human breast tumors are involved in spontaneous metastases in orthotopic mouse models. Proc. Natl. Acad. Sci. U.S.A. 107, 18115-18120.

Liu, S., Ginestier, C., Ou, S. J., Clouthier, S. G., Patel, S. H., Monville, F., et al. (2011). Breast cancer stem cells are regulated by mesenchymal stem cells through cytokine networks. Cancer Res. 71, 614-624.

Lou, H., and Dean, M. (2007). Targeted therapy for cancer stem cells: the patched pathway and $\mathrm{ABC}$ transporters. Oncogene 26, 1357-1360.

Louie, E., Nik, S., Chen, J. S., Schmidt, M., Song, B., Pacson, C., et al. (2010). Identification of a stem-like cell population by exposing metastatic breast cancer cell lines to repetitive cycles of hypoxia and reoxygenation. Breast Cancer Res. 12, R94.

Mani, S. A., Guo, W., Liao, M. J., Eaton, E. N., Ayyanan, A., Zhou, A. Y., et al. (2008). The epithelial-mesenchymal transition generates cells with properties of stem cells. Cell 133, 704-715.

Marotta, L. L., and Polyak, K. (2009). Cancer stem cells: a model in the making. Curr. Opin. Genet. Dev. 19, 44-50.

Meltzer, A. (1990). Dormancy and breast cancer. J. Surg. Oncol. 43, 181-188.

Micalizzi, D. S., Farabaugh, S. M. and Ford, H. L. (2010). Epithelialmesenchymal transition in cancer: parallels between normal development and tumor progression. J. Mammary Gland Biol. Neoplasia 15 117-134.

Moitra, K., Lou, H., and Dean, M. (2011). Multidrug efflux pumps and cancer stem cells: insights into multidrug resistance and therapeutic development. Clin. Pharmacol. Ther. 89, 491-502.
Nauseef, J. T., and Henry, M. D. (2011). Epithelial-to-mesenchymal transition in prostate cancer: paradigm or puzzle? Nat. Rev. Urol. 8, 428-439.

Newman, B., Liu, Y., Lee, H. F., Sun, D., and Wang, Y. (2012). HSP90 inhibitor 17-AAG selectively eradicates lymphoma stem cells. Cancer Res. 72, 4551-4561.

O’Brien, C. A., Kreso, A., and Dick, J. E. (2009). Cancer stem cells in solid tumors: an overview. Semin. Radiat. Oncol. 19, 71-77.

Oka, H., Shiozaki, H., Kobayashi, K., Inoue, M., Tahara, H., Kobayashi, T., et al. (1993). Expression of Ecadherin cell adhesion molecules in human breast cancer tissues and its relationship to metastasis. Cancer Res. 53, 1696-1701.

Oltean, S., Sorg, B. S., Albrecht, T., Bonano, V. I., Brazas, R. M., Dewhirst, M. W., et al. (2006). Alternative inclusion of fibroblast growth factor receptor 2 exon IIIc in Dunning prostate tumors reveals unexpected epithelial mesenchymal plasticity. Proc. Natl. Acad. Sci. U.S.A. 103, 14116-14121.

Pajonk, F., Vlashi, E., and McBride, W. H. (2010). Radiation resistance of cancer stem cells: the 4 R's of radiobiology revisited. Stem Cells 28, 639-648.

Pardal, R., Clarke, M. F., and Morrison, S. J. (2003). Applying the principles of stem-cell biology to cancer. Nat. Rev. Cancer 3, 895-902.

Park, S. M., Gaur, A. B., Lengyel, E., and Peter, M. E. (2008). The miR200 family determines the epithelial phenotype of cancer cells by targeting the E-cadherin repressors ZEB1 and ZEB2. Genes Dev. 22, 894-907.

Park, Y., and Gerson, S. L. (2005). DNA repair defects in stem cell function and aging. Annu. Rev. Med. 56, 495-508.

Patel, S. A., Meyer, J. R., Greco, S. J., Corcoran, K. E., Bryan, M., and Rameshwar, P. (2010). Mesenchymal stem cells protect breast cancer cells through regulatory $\mathrm{T}$ cells: role of mesenchymal stem cell-derived TGFbeta. J. Immunol. 184, 5885-5894.

Potten, C. S., Owen, G., and Booth, D. (2002). Intestinal stem cells protect their genome by selective segregation of template DNA strands. J. Cell Sci. 115, 2381-2388.

Raza, A., Franklin, M. J., and Dudek, A. Z. (2010). Pericytes and vessel maturation during tumor angiogenesis and metastasis. Am. J. Hematol. 85, 593-598.

Reya, T., Morrison, S. J., Clarke, M. F., and Weissman, I. L. (2001). Stem cells, cancer, and cancer stem cells. Nature 414, 105-111. 
Rich, J. N. (2007). Cancer stem cells in radiation resistance. Cancer Res. 67, 8980-8984.

Saharinen, P., Eklund, L., Pulkki, K., Bono, P., and Alitalo, K. (2011). VEGF and angiopoietin signaling in tumor angiogenesis and metastasis. Trends Mol. Med. 17, 347-362.

Said, N. A., and Williams, E. D. (2011). Growth factors in induction of epithelial-mesenchymal transition and metastasis. Cells Tissues Organs 193, 85-97.

Seton-Rogers, S. (2011). Cancer stem cells. VEGF promotes stemness. Nat. Rev. Cancer 11, 831.

Shi, X., Zhang, Y., Zheng, J., and Pan, J. (2012). Reactive oxygen species in cancer stem cells. Antioxid. Redox Signal. 16, 1215-1228.

Shinin, V., Gayraud-Morel, B., Gomes, D., and Tajbakhsh, S. (2006). Asymmetric division and cosegregation of template DNA strands in adult muscle satellite cells. Nat. Cell Biol. 8, 677-687.

Siemens, H., Jackstadt, R., Hunten, S., Kaller, M., Menssen, A., Gotz, U., et al. (2011). miR-34 and SNAIL form a double-negative feedback loop to regulate epithelial-mesenchymal transitions. Cell Cycle 10, 4256-4271.

Steeg, P. S. (2006). Tumor metastasis: mechanistic insights and clinical challenges. Nat. Med. 12, 895-904.

Suzuki, M., Mose, E. S., Montel, V., and Tarin, D. (2006). Dormant cancer cells retrieved from metastasisfree organs regain tumorigenic and metastatic potency. Am. J. Pathol. 169, 673-681.

Takahashi, Y., and Mai, M. (2005). Antibody against vascular endothelial growth factor (VEGF) inhibits angiogenic switch and liver metastasis in orthotopic xenograft model with site-dependent expression of VEGF. J. Exp. Clin. Cancer Res. 24, 237-243.

Tarin, D., Price, J. E., Kettlewell, M. G. Souter, R. G., Vass, A. C., and Crossley, B. (1984). Mechanisms of human tumor metastasis studied in patients with peritoneovenous shunts. Cancer Res. 44, 3584-3592.

Todaro, M., Alea, M. P., Di Stefano, A. B., Cammareri, P., Vermeulen, L. Iovino, F., et al. (2007). Colon cancer stem cells dictate tumor growth and resist cell death by production of interleukin-4. Cell Stem Cell 1, 389-402.

Tsuji, T., Ibaragi, S., and $\mathrm{Hu}, \mathrm{G}$. F. (2009). Epithelial-mesenchymal transition and cell cooperativity in metastasis. Cancer Res. 69, 71357139.

Tsuji, T., Ibaragi, S., Shima, K., Hu, M. G., Katsurano, M., Sasaki, A., et al. (2008). Epithelial-mesenchymal transition induced by growth suppressor p12CDK2-AP1 promotes tumor cell local invasion but suppresses distant colony growth. Cancer Res. 68, 10377-10386.

Uhr, J. W., Scheuermann, R. H., Street, N. E., and Vitetta, E. S. (1997). Cancer dormancy: opportunities for new therapeutic approaches. Nat. Med. 3, 505-509.

Vincan, E., Brabletz, T., Faux, M. C., and Ramsay, R. G. (2007a). A human three-dimensional cell line model allows the study of dynamic and reversible epithelialmesenchymal and mesenchymalepithelial transition that underpins colorectal carcinogenesis. Cells Tissues Organs 185, 20-28.

Vincan, E., Darcy, P. K., Farrelly, C. A., Faux, M. C., Brabletz, T., and Ramsay, R. G. (2007b). Frizzled-7 dictates three-dimensional organization of colorectal cancer cell carcinoids. Oncogene 26, 2340-2352.

Visvader, J. E., and Lindeman, G. J. (2008). Cancer stem cells in solid tumours: accumulating evidence and unresolved questions. Nat. Rev. Cancer 8, 755-768.

Wang, S., Yang, D., and Lippman, M. E. (2003). Targeting Bcl-2 and Bcl$\mathrm{XL}$ with nonpeptidic small-molecule antagonists. Semin. Oncol. 30, 133-142.

Wang, Z., Li, Y., and Sarkar, F. H (2010). Signaling mechanism(s) of reactive oxygen species in epithelialmesenchymal transition reminiscent of cancer stem cells in tumor progression. Curr. Stem Cell Res. Ther 5, 74-80.

Weigelt, B., Peterse, J. L., and van 't Veer, L. J. (2005). Breast cancer metastasis: markers and models. Nat. Rev. Cancer 5, 591-602.

Weiss, L. (1990). Metastatic inefficiency. Adv. Cancer Res. 54, 159-211.

Weiss, L. (1992). Comments on hematogenous metastatic patterns in humans as revealed by autopsy. Clin. Exp. Metastasis 10, 191-199.

Wu, K. J. (2011). Direct activation of Bmil by Twistl: implications in cancer stemness, epithelialmesenchymal transition, and clinical significance. Chang Gung Med. J. 34, 229-238.

Wu, K. J., and Yang, M. H. (2011) Epithelial-mesenchymal transition and cancer stemness: the Twist1Bmil connection. Biosci. Rep. 31, 449-455.

Yang, J., Mani, S. A., Donaher, J. L., Ramaswamy, S., Itzykson, R. A., Come, C., et al. (2004). Twist, a master regulator of morphogenesis, plays an essential role in tumor metastasis. Cell 117, 927-939.
Yao, D., Dai, C., and Peng, S. (2011). Mechanism of the mesenchymalepithelial transition and its relationship with metastatic tumor formation. Mol. Cancer Res. 9, 1608-1620.

Yates, C. C., Shepard, C. R., Stolz, D. B., and Wells, A. (2007). Co-culturing human prostate carcinoma cells with hepatocytes leads to increased expression of E-cadherin. Br. J. Cancer 96, 1246-1252.

Yilmaz, M., and Christofori, G. (2009). EMT, the cytoskeleton, and cancer cell invasion. Cancer Metastasis Rev. $28,15-33$.

Yoo, M. H., and Hatfield, D. L. (2008). The cancer stem cell theory: is it correct? Mol. Cells 26, 514-516.

Conflict of Interest Statement: The authors declare that the research was conducted in the absence of any commercial or financial relationships that could be construed as a potential conflict of interest.

Received: 25 July 2012; accepted: 02 October 2012; published online: 23 October 2012.

Citation: Patel P and Chen EI (2012) Cancer stem cells, tumor dormancy, and metastasis. Front. Endocrin. 3:125. doi: 10.3389/fendo.2012.00125

This article was submitted to Frontiers in Cancer Endocrinology, a specialty of Frontiers in Endocrinology.

Copyright (C) 2012 Patel and Chen. This is an open-access article distributed under the terms of the Creative Commons Attribution License, which permits use, distribution and reproduction in other forums, provided the original authors and source are credited and subject to any copyright notices concerning any thirdparty graphics etc. 